\title{
AN IMAGING OBSERVATION OF SN 1987A AT GAMMA-RAY ENERGIES
}

\author{
W. R. Cook, D. M. Palmer, T. A. Prince, S. M. Schindler, C. H. Stark, and E. C. Stone \\ California Institute of Technology \\ Received 1988 May 12 ; accepted 1988 August 24
}

\begin{abstract}
The Caltech imaging $\gamma$-ray telescope was launched by balloon from Alice Springs, NT, Australia for observations of SN 1987A during the period 1987 November $18.60-18.87$ UT. The preliminary results presented here are derived from $8200 \mathrm{~s}$ of instrument live time on the supernova and $2500 \mathrm{~s}$ on the Crab Nebula and pulsar at a float altitude of $36 \mathrm{~km}$. We have obtained the first images of the SN 1987A region at $\gamma$-ray energies confirming that the bulk of the $\gamma$-ray emission comes from the supernova and not from LMC X-1. We compare our flux measurements to recent predictions concerning the distribution of ${ }^{56} \mathrm{Co}$ in the supernova ejecta and find the data to be consistent with models invoking moderate mixing of core material into the envelope.

Subject headings: gamma rays: general — gamma rays: spectra — stars: individual (SN 1987A) stars: supernovae
\end{abstract}

\section{INTRODUCTION}

The occurrence of SN 1987A in the Large Magellanic Cloud has provided the first opportunity to study the hard X-ray and $\gamma$-ray emission from a Type II supernova. There have been numerous predictions of the $\gamma$-ray line flux from Type II events (cf. Bodansky, Clayton, and Fowler 1968; Clayton, Colgate, and Fishman 1969; and Colgate and McKee 1969); in contrast, the continuum flux has received less attention until the recent SN 1987A event. Initial hard X-ray and $\gamma$-ray detections of SN 1987A were made by several satellite instruments during 1987 July-August (Sunyaev et al. 1987, 1988; Dotani et al. 1987; and Matz et al. 1988). Further observations were performed by several balloon-borne instruments during the period 1987 October-December. This Letter reports on one of these--the first imaging observation of the supernova at $\gamma$-ray energies.

The observations were performed with the Caltech GammaRay Imaging Payload (GRIP), a balloon-borne coded-aperture telescope sensitive to radiation in the energy range from 30 $\mathrm{keV}$ to $10 \mathrm{MeV}$ (Althouse et al. 1985). The instrument employs a rotating hexagonal-celled uniformly redundant array (URA) and a $5 \mathrm{~cm}$ thick by $41 \mathrm{~cm}$ diameter position sensitive NaI scintillation detector to image a $14^{\circ}$ diameter field of view with 1.1 angular resolution. The mask cell size used in the flight on 1987 November 18 was $4.8 \mathrm{~cm}$, and the separation of the mask and detector was $2.5 \mathrm{~m}$. The instrument has flown twice previously (Althouse et al. 1987), including a flight in 1987 May from Australia to observe SN 1987A. No $\gamma$-ray or hard X-ray emission was detected in the 1987 May observation (Cook et al. 1987).

\section{IMAGES}

The analysis of data from a coded-aperture detector involves the detection of a source and determination of its position, followed by detailed analysis of the spectral characteristics of the excess flux at the position of the source. We will briefly summarize the analysis procedure, which is described in more detail by Cook et al. (1984).

Photon event positions are obtained using a positiondetermination algorithm derived from laboratory calibrations.
For a given orientation of the coded-aperture mask, each event is back-projected onto the sky through the center of each mask cell to create a sky image, contributing a positive count to the sky image if a cell is open, a negative count if the cell is obscured with a lead block, and, for technical reasons, zero counts if the cell is the center cell - of the hexagonal URA pattern. Because the number of open and blocked cells is equal, the sum of counts in the sky image is zero.

The data from different mask orientations (typically 360 in our analysis) are then added by rotating the individual sky images to a common orientation; this addition also implements the mask-antimask background subtraction for the hexagonal URAs. Finally, the resulting image is smoothed by convolution with an angular point-spread function derived from the detector position-determination point-spread function and the size and shape of the mask cells. This yields an image which is statistically optimal for measurements of the flux and location of point sources (Cook et al. 1984).

The convolved image is calibrated in terms of the statistical significance of the excess number of counts at a given image position. The statistics of the image are the Poisson statistics of the count difference between events passing through open and blocked mask cells and are dependent only on the total number of events and the angular point-spread function employed in the convolution.

Given a sky image over a broad energy range, the count distribution is scanned for significant excesses. If a source is detected, the source spectrum is determined from images for several different energy bands. The flux measurement for each energy band is derived from the count excess (positive or negative) at the position of the source, taking into account the efficiency factor appropriate for the convolving function, the observation live time, the detector's effective area, and the attenuation of photons in the atmosphere and instrument.

Figure 1 shows an image of the SN 1987A region for energies between 40 and $1300 \mathrm{keV}$. The image is a composite of data from four individual supernova observation periods, which alternated with four calibration observations of the Crab Nebula and pulsar. The supernova image fields were aligned by using residual pointing corrections determined from the 


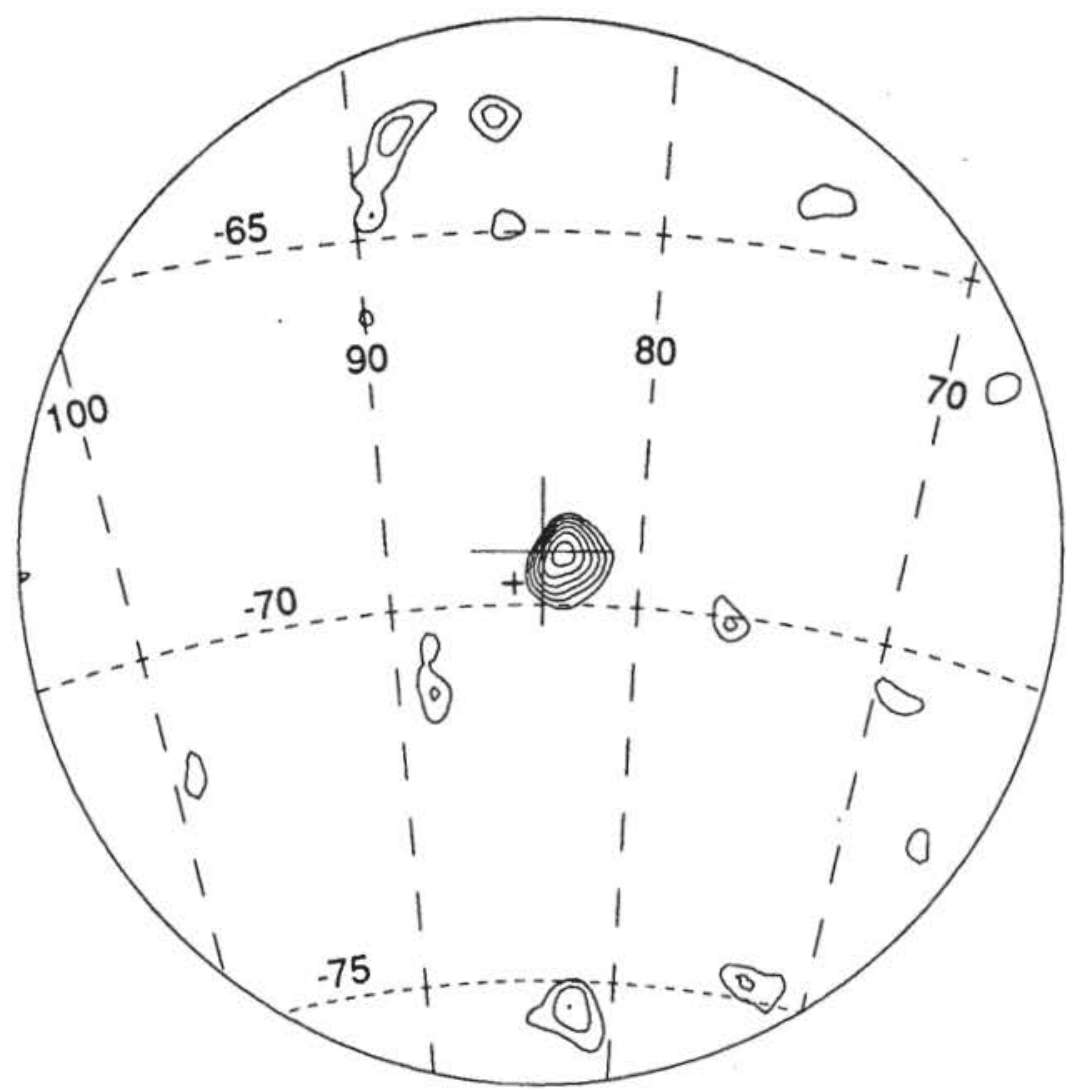

Fic. 1.-Image of the SN $1987 \mathrm{~A}$ region from 40 to $1300 \mathrm{keV}$ covering a $14^{\circ}$ field of view. Right ascension (vertical lines) and declination (horizontal lines) are indicated. The contours indicate the number of excess counts in a given direction calibrated in units of the statistical significance of the excess, with contours beginning at the $2 \sigma$ level and spaced by $0.5 \sigma$ intervals. The large cross indicates the expected position of SN 1987A. The small cross indicates the expected position of LMCX-1.

Crab observations. Flux is detected at a nominal $4.9 \sigma$ level from within 0.28 of the expected position of SN 1987A. The $1 \sigma$ error in the peak location is estimated to be 0.14 in the elevation direction and 0.27 in the azimuthal direction. The errors include both statistical error and an estimate of residual systematic errors determined from the Crab observations. The deviation from the expected position is in the azimuthal direction, and the larger error in this direction may be due to deviations between the actual and the assumed magnetic field direction used as a pointing reference during flight. In the elevation direction, which is approximately aligned with the declination axis in these observations, the peak centroid is consistent with the position of the supernova.

Because these are the first imaging observations of $\mathrm{SN}$ $1987 \mathrm{~A}$ at energies greater than $30 \mathrm{keV}$, it is valuable to investigate other astrophysical sources as the possible origin of the gamma-ray emission. The one nearby object most likely to be a contaminating source is the black hole candidate LMC X-1 at $\alpha(1950)=85^{\circ} .03$ and $\delta(1950)=-69^{\circ} .77$, which is 0.60 from SN $1987 \mathrm{~A}$ at $\alpha(1950)=83^{\circ} .96$ and $\delta(1950)=-69^{\circ} .30$. It is conceivable that LMC X-1 could have a hard, time-variable, nonthermal tail of emission extending up to $\mathrm{MeV}$ energies, much like $\mathrm{Cyg} X-1$. In fact, LMC X-1 is known to be a strong source at $X$-ray energies, and there is some uncertainty over its possible contribution to the X-ray flux reported from SN 1987A (Sunyaev et al. 1987; Dotani et al. 1987).

Because of the azimuthal uncertainty in our aspect determination at the current stage of analysis, it is impossible to eliminate LMC X-I entirely as a contributor to the gamma-ray flux.
However, because LMC X-1 is 0.5 away from SN 1987A in the elevation direction, and because the $1 \sigma$ error for the position determination in the elevation direction is 0.14 , the chance that LMC X-1 is the sole source of emission is less than $0.1 \%$. For the remainder of this Letter, we assume that the supernova is the sole source of excess photons in the peak in the image of Figure 1.

\section{SPECTRA}

Measurements of the $\gamma$-ray flux from SN 1987A for selected energy intervals are listed in Table 1 and shown in Figure 2. The flux measurements were derived from the number of excess counts in the specified energy interval at the peak position

TABLE 1

SN 1987A FluX MEAsUrements

\begin{tabular}{|c|c|c|}
\hline $\begin{array}{l}\text { Energy Interval } \\
\text { (keV) }\end{array}$ & $\begin{array}{c}\text { Differential Flux } \\
\left(10^{-3} \mathrm{~cm}^{-2} \mathrm{~s}^{-1} \mathrm{keV}^{-1}\right)\end{array}$ & $\begin{array}{l}\text { Integral Flux } \\
\left(10^{-3} \mathrm{~cm}^{-2} \mathrm{~s}^{-1}\right)\end{array}$ \\
\hline 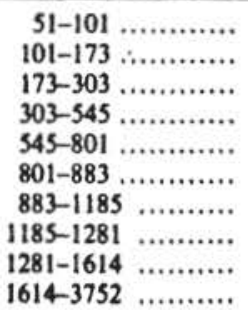 & $\begin{array}{l}4.73 \pm 2.17 \\
2.29 \pm 0.84 \\
1.04 \pm 0.57 \\
0.32 \pm 0.47 \\
0.33 \pm 0.37 \\
1.32 \pm 0.69 \\
0.62 \pm 0.38 \\
1.15 \pm 0.66 \\
0.08 \pm 0.43 \\
<0.37(3 \sigma)\end{array}$ & $\begin{array}{c}\ldots \\
\ldots \\
\ldots \\
\ldots \\
\ldots \\
1.08 \pm 0.56^{\circ} \\
\ldots \\
1.11 \pm 0.64^{\circ} \\
\ldots \\
\ldots\end{array}$ \\
\hline
\end{tabular}

- Includes both continuum and line flux; see text for line flux estimatea 


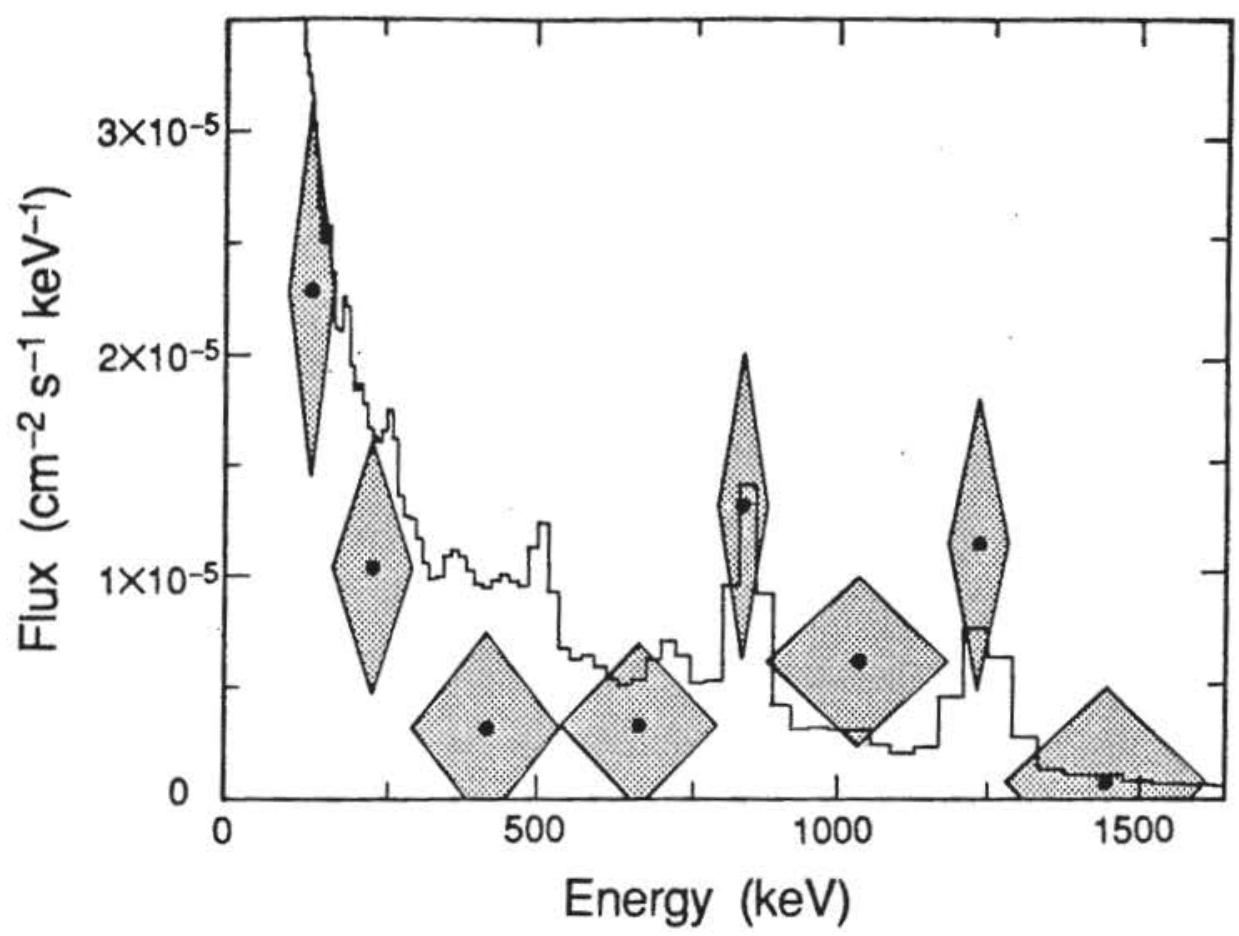

Fig. 2.-Flux measurements compared with the result of a Monte Carlo calculation of the expected flux on day 250 of the supernova (Pinto and Woosley 1988). The measurements are indicated by diamonds with the width indicating the energy interval of the measurement. The Monte Carlo histogram has been convolved with a Gaussian representation of the instrumental energy resolution.

determined from Figure 1. To obtain the overall characteristics of the spectrum, the data were fitted to a power-law differential continuum flux, $F=K(E / 100 \mathrm{keV})^{\alpha}$, over the energy range 51-1614 keV. A very hard power-law index, $\alpha=-0.9$, and a flux normalization, $K=2.8 \times 10^{-3}\left(\mathrm{~cm}^{2} \mathrm{~s} \mathrm{keV}\right)^{-1}$, were obtained. For comparison, the same type of analysis for the Crab yielded a flux of $4.4 \times 10^{-4}(E / 100 \mathrm{keV})^{-2}\left(\mathrm{~cm}^{2} \mathrm{~s} \mathrm{keV}\right)^{-1}$ between 40 and $500 \mathrm{keV}$.

It is of interest to estimate the flux at the energies of the prominent $\gamma$-ray lines expected from ${ }^{56} \mathrm{Co}$ decay. Table 1 gives flux values for intervals including $847 \mathrm{keV}$ and $1238 \mathrm{keV}$. The widths of the intervals are chosen such that $89 \%$ and $84 \%$, respectively, of the narrow line flux is contained in the intervals.

To estimate the amount of possible ${ }^{56} \mathrm{Co}$ line flux enhancement above the continuum level, we have performed a minimum- $\chi^{2}$ fit to our measurements in the energy range 545$1614 \mathrm{keV}$. For the fitting function we chose a straight-line continuum with gamma-ray line contributions described by two Gaussian functions centered at 847 and $1238 \mathrm{keV}$, having widths fixed at the instrumental energy resolution of $7 \%$ and $6.5 \%$ FWHM, respectively. The flux level and slope of the continuum contribution, as well as the line flux levels, were varied to minimize $\chi^{2}$. The resulting minimum occurred at a reduced $x^{2}$ of 0.92 (with 26 degrees of freedom) for values of the 847 and $1238 \mathrm{keV}$ line fluxes of $0.5 \pm 0.6 \times 10^{-3}$ and $1.3 \pm 0.8 \times 10^{-3} \mathrm{~cm}^{-2} \mathrm{~s}^{-1}$, respectively.

The results reported here are preliminary. Most conceivable systematic errors, in particular, pointing uncertainties, would tend to yield an absolute measured flux somewhat lower than the true flux. We note that the estimate of the Crab spectrum given above is $\sim 20 \%-40 \%$ below other measurements at 100 $\mathrm{keV}$ (Jung 1986) and may indicate an overall systematic underestimate of the flux. However, the earlier measurements were made with nonimaging instruments which may overestimate the flux, and there is also the possibility of time variability.

\section{DISCUSSION}

The results from Table 1 should be compared to other measurements of the hard $\mathrm{X}$-ray and $\gamma$-ray flux made during the period of 1987 August through November. These include results using the Mir-Kvant observatory (Sunyaev et al. 1987, 1988), the Ginga satellite (Dotani et al. 1987), and the Solar Maximum Mission (SMM) observatory (Matz et al. 1988). Our continuum flux measurements on 1987 November 18 at energies from 51 to $173 \mathrm{keV}$ are consistent with the flux of approximately $3.0 \times 10^{-5}\left(\mathrm{~cm}^{2} \mathrm{~s} \mathrm{keV}\right)^{-1}$ at $100 \mathrm{keV}$ measured by the Mir-Kvant observatory during 1987 November (Sunyaev et al. 1988). The integral fluxes for the $847 \mathrm{keV}$ and $1238 \mathrm{keV}$ regions from Table 1 include both line and continuum contributions. The fits to the lines discussed above yielded values of $0.5 \pm 0.6 \times 10^{-3}$ and $1.3 \pm 0.8 \times 10^{-3} \mathrm{~cm}^{-2} \mathrm{~s}^{-1}$ for 847 and $1238 \mathrm{keV}$, respectively, consistent with the $\gamma$-ray line fluxes reported by the SMM instrument averaged over the 1987 August through October period: $1.0 \pm 0.25 \times 10^{-3} \mathrm{~cm}^{-2} \mathrm{~s}^{-1}$ at $847 \mathrm{keV}$ and $0.6 \pm 0.2 \times 10^{-3} \mathrm{~cm}^{-2} \mathrm{~s}^{-1}$ at $1238 \mathrm{keV}$. Our measurements should also be compared to the forthcoming results from three other balloon-borne instruments. These include the hard X-ray measurements from 45 to $200 \mathrm{keV}$ of Wilson et al. (1988) on 1987 October 29-30, and the highresolution $\gamma$-ray line measurements of Sandie et al. (1988) and Mahoney et al. (1988) on 1987 October 29-30 and 1987 December 6, respectively.

A likely origin of the observed hard X-ray and $\gamma$-ray flux is the decay of ${ }^{56} \mathrm{Co}$ which is a decay product of ${ }^{56} \mathrm{Ni}$ synthesized in the supernova explosion (Bodansky, Clayton, and Fowler 1968; Clayton, Colgate, and Fishman 1969; Colgate and 
McKee 1969). The ${ }^{36}$ Co decays are expected to yield both line emission and a continuum of Compton-degraded photons. Approximately $0.075 M_{\odot}$ of ${ }^{36} \mathrm{Ni}$ was synthesized in the initial explosion, as determined from the decay of the bolometric luminosity of the supernova (Woosley 1988). Results from Table 1 are compared with recent theoretical predictions in Figure 2. The continuous histogram is based on a Monte Carlo calculation of the expected photon flux on day 250 (18 days before our observation), taken from Pinto and Woosley (1988). Similar calculations have been carried out by other authors (Itoh et al. 1987; McCray, Shull, and Sutherland 1987; Arnett and Fu 1989; Ebisuzaki and Shibazaki 1988; Fu and Arnett 1989; Shibazaki and Ebisuzaki 1988; Xu et al. 1988); the calculation in Figure 2 is an example of models which incorporate mixing to explain the early turn-on of X-rays and $\gamma$-rays seen by the Mir-Kvant, Ginga, and SMM instruments. The model assumes a $6 M_{\odot} \mathrm{He}$ core, a $10 M_{\odot}$ hydrogen envelope,
$1.3 \times 10^{51}$ ergs explosion kinetic energy, and a moderate degree of mixing of ${ }^{56} \mathrm{Co}$ out into the helium envelope. Our flux measurements shown in Figure 2 are in qualitative agreement with the calculations. In particular, when the flux in the regions of the lines is compared to the continuum flux measurements, the results suggest a flux excess in the region of the expected $\gamma$-ray lines from ${ }^{56} \mathrm{Co}$ decay as predicted by the Monte Carlo calculations for this time period.

We acknowledge the important contributions to the development of the GRIP telescope made by W. Althouse, D. Burke, A. Cummings, M. Finger, J. Weger, and the personnel of Central Engineering Services at the California Institute of Technology. We thank the personnel of the National Scientific Balloon Facility and the NASA Wallops Flight Facility for their excellent balloon launch support. This work was supported by NASA grant NGR 05-002-160.
Althouse, W. E, et al. 1985, Proc. 19th Int. Cosmic Ray Conf. (La Jolla) (NASA CP-2376), 3, 299.

Althouse, W. E., et al. 1987, Proc. 20th Int. Cosmic Ray Conf. (Moscow), 1, 84.

Arnett, W. D., and Fu, A. 1989, Ap. J., in press.

Bodansky, D., Clayton, D. D., and Fowler, W. A. 1968, Ap. J. Suppl., 16, 299.

Clayton, D. D., Colgate, S. A., and Fishman, G. J. 1969, Ap. J., 155, 75.

Colgate, S. A., and McKee, C. 1969, Ap. J., 157, 623.

Cook, W., Palmer, D., Prince, T., Schindler, S., and Stone, E. 1987, IAU Circ., 4400 .

Cook, W. R., Finger, M., Prince, T. A., and Stone, E. C. 1984, IEEE Trans. Nucl. Sci, NS-31, 771 .

Dotani, T., et al. 1987, Nature, 330, 230.

Ebisuzaki, T., and Shibazaki, N. 1988, Ap. J. (Letters), 327, L5.

Fu, A., and Amett, W. D. 1989, Ap. J., in press.

Itoh, M., Kumagai, S., Shigeyama, T., Nomoto, K., and Nishimura, J. 1987, Nature, 330,233

Jung, G. V. 1986, Ph.D. thesis, University of California at San Diego.

\section{REFERENCES}

Mahoney, W. A., Varnell, L. S., Jacobson, A. S., Ling, J. C., Radocinski, R. G., and Wheaton, Wm. A. 1988, Ap. J. (Letters), 334, L81.

Matz S. M., Share, G. H., Leising, M. D., Chupp, E. L., Vestrand, W. T. Purcell, W. R., Strickman, M. S., and Reppin, C. 1988, Nature, 331, 416.

McCray, R., Shull, J. M., and Sutherland, P. 1987, Ap. J. (Letters), 317, L73.

Pinto, P. A., and Woosley, S. E. 1988, Nature, 333, 534.

Sandie, W. G., Nakano, G. H., Chase, L. F. Jr., Meegan, C. A., Wilson, R. B., Paciesas, W. S., and Lasche, G. P. 1988, Ap. J. (Letters), 334, L91.

Shibazaki, N., and Ebisuzaki, T. 1988, Ap. J. (Letters), 327, L9.

Sunyaev, R., et al. 1987, Nature, 330, 227.

Sunyaev, R., et al. 1988, Soviet Astr. Letters, Vol. 15, No. 7, p. 579.

Wilson, R., Fishman, G., Meegan, C., Paciesas, W., and Pendleton, G. 1988, in Nuclear Spectroscopy of Astrophysical Sources, ed. N. Gehrels and G. Share (New York: AIP), in press.

Woosley, S. 1988, Ap. J., 330, 218.

Xu, Y., Sutherland, P., McCray, R., and Ross, R. R. 1988, Ap. J., 327, 197.

Walter R. Cook, David M. Palmer, Thomas A. Prince, Stephen M. Schindler, Christopher H. Starr, and Edward C. Stone: 220-47, California Institute of Technology, Pasadena, CA 91125 